\title{
Eficácia e seletividade de herbicidas do grupo das imizadolinonas aplicados em arroz irrigado ${ }^{1}$
}

\section{Efficacy and selectivity of imidazolinone herbicides applied in flooded rice}

\author{
Leandro Galon ${ }^{2}$; Sergio Guimarães ${ }^{3}$; Giovane Matias Burg ${ }^{3}$; Germani Concenço ${ }^{4}$; Antonio Carlos \\ Rampelotto Filho ${ }^{3}$; Anderson Moraes de Lima ${ }^{3}$; Juliana Gomes Belarmino ${ }^{3}$
}

Resumo - Objetivou-se com o trabalho avaliar a eficiência no controle do arroz-vermelho e a seletividade ao arroz irrigado pela aplicação de herbicidas do grupo químico das imidazolinonas. O delineamento experimental utilizado foi o de blocos ao acaso, com quatro repetições, arranjado em esquema fatorial 4 x 4 . O fator A foi composto por herbicidas e o B pelas doses dos mesmos. Os herbicidas e doses foram: imazethapyr + imazapic $[(0,00 ; 37,50+12,50 ; 75,00+25,00$ e $\left.150,00+50,00 \mathrm{~g} \mathrm{ha}^{-1}\right)+$ Dash $\left.0,5 \% \mathrm{v} / \mathrm{v}\right]$, imazapyr + imazapic $[(0,00 ; 36,75+12,25 ; 73,50+$ 24,50; 147,00 + 49,00 $\left.\mathrm{g} \mathrm{ha}^{-1}\right)+$ Dash 0,5\% v/v], imazethapyr $\left(0 ; 75,00 ; 150,00\right.$ e 300,00 $\left.\mathrm{g} \mathrm{ha}^{-1}\right) \mathrm{e}$ imazapic $\left(0,00 ; 12,25 ; 24,50\right.$ e $49,00 \mathrm{~g} \mathrm{ha}^{-1}+$ Dash $\left.0,5 \% \mathrm{v} / \mathrm{v}\right)$. As variáveis avaliadas foram: controle do arroz-vermelho efetuado aos 14, e 28 dias após a aplicação dos tratamentos (DAT) e na pré-colheita do arroz; e a intoxicação ao arroz, determinada aos 07, 14 e 21 DAT. As misturas formuladas comercialmente compostas de imazapyr + imazapic na dose recomendada $(73,5+$ $\left.24,5 \mathrm{~g} \mathrm{ha}^{-1}\right)$ e de imazethapyr + imazapic no dobro da dose recomendada $\left(150+50 \mathrm{~g} \mathrm{ha}^{-1}\right)$ foram eficientes para o controle de arroz-vermelho. Estas mesmas misturas também foram seletivas a cultivar Puitá Inta-CL, independentemente das doses aplicadas dos produtos. Os herbicidas imazethapyr e imazapic, não registrados para o controle de arroz-vermelho, não apresentaram controle satisfatório desta espécie daninha.

Palavras-chaves: Inibidores de ALS, Oryza sativa, controle químico

Abstract - This study aimed to evaluate the control of red rice and the selectivity to commercial mutant rice varieties under application of imidazolinone herbicides. The experiment was installed in completely randomized blocks design, under factorial scheme $4 \times 4$, with four replications. Factor A was composed by the herbicides and Factor B by doses of these herbicides. The herbicides and doses were: imazethapyr + imazapic $[(0.00 ; 37.50+12.50 ; 75.00+25.00$ and $\left.150.0+50.00 \mathrm{~g} \mathrm{ha}^{-1}\right)+$ Dash $\left.0.5 \mathrm{v} \mathrm{v}^{-1}\right]$, imazapyr + imazapic $[(0.00 ; 36.75+12.25 ; 73.50+24.50$ and $\left.147.00+49.00 \mathrm{~g} \mathrm{ha}^{-1}\right)+$ Dash $\left.0.5 \mathrm{v} \mathrm{v}^{-1}\right]$, imazethapyr $\left(0.00 ; 75.00 ; 150.00\right.$ and $\left.300.00 \mathrm{~g} \mathrm{ha}^{-1}\right)$ and imazapic $\left(0.00 ; 12.25 ; 24.50\right.$ and $49.00 \mathrm{~g} \mathrm{ha}^{-1}+$ Dash $\left.0.5 \mathrm{v} \mathrm{v}^{-1}\right)$. The control of red rice was evaluated 14 and 28 days after treatment (DAT), as well as at the pre-harvest; toxicity to

\footnotetext{
${ }^{1}$ Recebido para publicação em 12/03/2012 e aceito em 27/09/2012.

${ }^{2}$ Professor da Universidade Federal da Fronteira Sul (UFFS), Campus Erechim, Curso de Agronomia, Av. Dom João Hoffmann, 313, CEP.: 99700-000, Erechim, RS. Email: <leandro.galon@uffs.edu.br>. (Autor para correspondência). ${ }^{3}$ Acadêmicos de Agronomia da Universidade Federal do Pampa (UNIPAMPA), Campus Itaqui, Rua Luiz Joaquim de Sá Britto, s/n, CEP: 97650-000, Itaqui, RS. E-mails: sergioguimaraessg@ hotmail.com; toninho.rf@ hotmail.com; agro.gio@hotmail.com; andersonmoraees@gmail.com e juzinha_belarmino@hotmail.com.

${ }^{4}$ Pesquisador em Herbologia da Embrapa Agropecuária Oeste (CPAO), BR 163, km 253,6 - Caixa Postal no 449, CEP.: 79804-970, Dourados, MS. E-mail: <germani@cpao.embrapa.br>.
} 
commercial rice was evaluated 7, 14 and 21 DAT. Commercially available mixtures of imazapic + imazapyr, at the recommended dose $\left(73.5+24.5 \mathrm{~g} \mathrm{ha}^{-1}\right)$, and imazethapyr + imazapic at twice the dose $\left(150.00+50.00 \mathrm{~g} \mathrm{ha}^{-1}\right)$ were efficient in controlling red rice. These same mixtures were also selective to the rice variety Puitá Inta-CL, independently of dose. Herbicides not registered for control of red rice, imazethapyr and imazapic, were not efficient in controlling red rice.

Keywords: ALS inhibitor, Oryza sativa, chemical control

\section{Introdução}

A cultura do arroz representa $8 \%$ da produção de cereais do Brasil, sendo que a Região Sul responde por aproximadamente $70 \%$ da produção nacional de grãos de arroz (SOSBAI, 2010). No Rio Grande do Sul (RS), o arroz ocupa uma área superior a um milhão de hectares, com uma produção que ultrapassa 8,2 milhões de toneladas e uma produtividade média de 7,6 $\mathrm{t} \mathrm{ha}^{-1}$ (CONAB, 2012).

Várias são as espécies de plantas daninhas que infestam as lavouras de arroz irrigado. Dentre elas, o arroz-daninho (arrozvermelho e preto) está entre as mais problemáticas e de difícil controle, por apresentar características morfológicas, fisiológicas e bioquímicas similares ao arroz irrigado (Sanchez-Olquin et al., 2007). O arrozdaninho demonstra elevada habilidade competitiva com a cultura, afetando diretamente o rendimento de grãos do arroz e a qualidade do produto colhido (Agostinetto et al., 2001). As perdas no rendimento de grãos ocasionadas por esta planta daninha ao competir com o arroz irrigado por luz, nutrientes e $\mathrm{CO}_{2}$ podem ser superiores a $20 \%$ (Marchezan et al., 2011) ou que uma única planta para cada $\mathrm{m}^{-2}$ pode reduzir em $755 \mathrm{~kg}$ $\mathrm{ha}^{-1}$ a produtividade da cultura (Ottis et al., 2005). Em países produtores de arroz irrigado estima-se que as perdas ocasionadas pela infestação do arroz-daninho é de cerca de US\$ $300 \mathrm{ha}^{-1}$ (Burgos et al., 2008). No Estado do RS perde-se, anualmente, cerca de 1,2 milhões de toneladas de grãos de arroz, valor equivalente a US\$ 360 milhões em função da competição do arroz-daninho nas lavouras orizícolas (Menezes, 1996).
O controle de arroz-vermelho por meio de herbicidas seletivos foi considerado prática inviável, pelo fato de o arroz cultivado e o arroz-daninho pertencerem à mesma espécie e apresentarem similaridades morfofisiológicas (Menezes et al., 2009), ou seja, aplicava-se o herbicida para controlar a planta daninha e ocasionava-se ao mesmo tempo a morte do arroz. Ressalta-se ainda que o controle de arroz-vermelho utilizando outros métodos apresenta eficiência limitada, sobretudo em grandes áreas de cultivo (Roso et al., 2010).

$\mathrm{Na}$ busca por alternativas para $\mathrm{o}$ controle do arroz-vermelho foram desenvolvidas cultivares de arroz tolerantes ao grupo de herbicidas das imidazolinonas, a partir de sementes de arroz da linhagem AS3510, sendo essas mutagenizadas com etilmetanossulfonato - EMS (Croughan, 1998). Os herbicidas pertencentes as imidazolinonas inibem a atividade das enzimas acetolactato sintase (ALS) e aceto-hidroxi sintase (AHAS) na rota de síntese dos aminoácidos de cadeia ramificada valina, leucina e isoleucina (Rizzardi et al., 2002).

Para que as alternativas de controle do arroz-vermelho sejam efetivas e viáveis, são necessárias avaliações de todos os aspectos do sistema produtivo, desde a eficiência de controle até seus possíveis efeitos no ambiente (Santos et al., 2007). Nesse contexto, a semeadura de genótipos tolerantes a herbicidas, aliado ao uso inadequado dos produtos, pode implicar em aspectos negativos relacionados à seleção de populações de arroz-vermelho resistentes. Além disso, existe o problema da permanência do grupo das imidazolinonas no sistema produtivo, pois esses herbicidas apresentam prolongada atividade residual no 
solo (Loux et al., 1989; Loux \& Reese, 1993), que pode variar de 31 a 410 dias para imazapic (Grymes, 1995) de 60 a 360 dias para o imazethapyr (Goetz, 1990) e de até 436 dias para imazapyr (Cox, 1996). Esta persistência no solo impede o cultivo de culturas como milho, sorgo ou azevém (Pinto et al., 2009a; 2009b; 2009c), girassol (Santos et al., 2012) dentre outras, em sucessão ao arroz irrigado ou mesmo a semeadura, em anos posteriores a aplicação, de cultivares de arroz que não são tolerantes aos produtos.

$\mathrm{Na}$ atualidade muitos produtores de arroz, principalmente da Fronteira Oeste do $\mathrm{RS}$, aplicam herbicidas pertencentes ao grupo químico das imidazolinonas, porém não recomendados à cultura. Esses produtos, na maioria das vezes provem de outros estados ou até mesmo de países vizinhos. Em função da tolerância de muitas variedades de arroz a esse grupo de herbicidas são utilizados para o controle de plantas daninhas, no entanto sem registro para esse fim em arroz e no Brasil. Esses herbicidas podem não controlar de modo adequado o arroz-vermelho, ou somente suprimir o desenvolvimento da planta daninha, com o passar do tempo poderá favorecer a ocorrência da resistência por pressão de seleção. Esses produtos podem ainda ocasionar maior intoxicação ao arroz se comparado aos recomendados à cultura, além de em muitos casos contaminarem o ambiente e persistirem no solo por maior tempo.

O presente trabalho tem como objetivo avaliar a eficiência no controle do arrozvermelho e a seletividade ao arroz irrigado pela aplicação de herbicidas do grupo químico das imidazolinonas.

\section{Material e Métodos}

O experimento foi conduzido a campo, no município de Itaqui-RS, no ano agrícola de 2010/11, em Plintossolo Háplico Distrófico (Embrapa, 2006), sendo preparado no sistema convencional de cultivo.
O delineamento experimental utilizado foi o de blocos ao acaso, com quatro repetições, arranjado os tratamentos em esquema fatorial $4 \times 4$. $\mathrm{O}$ fator $\mathrm{A}$ foi composto por herbicidas e o B pelas doses dos mesmos. Os herbicidas e doses foram: imazethapyr + imazapic - $[(0,00 ; 37,50+12,50 ; 75,00+$ 25,00 e $\left.150,00+50,00 \mathrm{~g} \mathrm{ha}^{-1}\right)+$ Dash $0,5 \%$ $\mathrm{v} / \mathrm{v}]$, imazapyr + imazapic $[(0,00 ; 36,75+$ 12,$\left.25 ; 73,50+24,50 ; 147,00+49,00 \mathrm{~g} \mathrm{ha}^{-1}\right)+$ Dash $0,5 \% \mathrm{v} / \mathrm{v}]$, imazethapyr $(0,00 ; 75,00$; 150,00 e $\left.300,00 \mathrm{~g} \mathrm{ha}^{-1}\right)$ e imazapic $(0,00$; 12,25; 24,50 e 49,00 $\mathrm{g} \mathrm{ha}^{-1}+$ Dash $\left.0,5 \% \mathrm{v} / \mathrm{v}\right)$. As doses utilizadas equivalem a 0,50, $100 \mathrm{e}$ $200 \%$ da recomenda dos herbicidas para o controle de arroz-daninho em lavouras de arroz irrigado.

A aplicação dos herbicidas foi efetuada com pulverizador costal de precisão, pressurizado a $\mathrm{CO}_{2}$, equipado com quatro pontas de pulverização tipo leque DG 110.02, mantendo-se pressão constante de $210 \mathrm{kPa}$ e velocidade de deslocamento de $3,6 \mathrm{~km} \mathrm{~h}^{-1}$, o que proporcionou a vazão de $150 \mathrm{~L} \mathrm{ha}^{-1}$ de calda. As condições no momento da aplicação foram: temperatura de $26{ }^{\circ} \mathrm{C}$, umidade relativa do ar de $72 \%$, solo seco e ventos de $3 \mathrm{~km} \mathrm{~s}^{-1}$. No momento da aplicação dos herbicidas o arroz encontrava-se no estádio de duas folhas a um perfilho e o arroz-vermelho de duas a quatro folhas, com uma população média de 100 plantas $\mathrm{m}^{-2}$. A dose recomendada de imazethapyr + imazapic para o controle de arroz-daninho em arroz irrigado é de 75,0 + $25,0 \mathrm{~g} \mathrm{ha}^{-1}$ e imazapic + imazapyr de 73,5 + $24,5 \mathrm{~g} \mathrm{ha}^{-1}$. Para os herbicidas imazethapyr e imazapic aplicou-se 150,0 e $24,5 \mathrm{~g} \mathrm{ha}^{-1}$, respectivamente, como sendo a dose utilizada pelos orizicultores da Fronteira Oeste do RS, como sendo $100 \%$.

Cada unidade experimental foi caracterizada por uma parcela de $11,05 \mathrm{~m}^{2}(5 \mathrm{x}$ $2,21 \mathrm{~m})$, semeadas com a cultivar Puitá IntaCL na densidade de $80 \mathrm{~kg} \mathrm{ha}^{-1}$, com 13 linhas de arroz no espaçamento de $0,17 \mathrm{~m}$. As avaliações de controle foram realizadas 
visualmente aos 14 e 28 dias após a aplicação dos tratamentos (DAT) e na pré-colheita da cultura, e as de intoxicação à cultura do arroz aos 7, 14 e 21 DAT, atribuindo-se a nota de zero $(0 \%)$ aos tratamentos com ausência de controle do arroz-vermelho ou intoxicação da cultura e a nota de cem (100\%) para controle total da planta daninha ou morte completa das plantas de arroz.

Os dados foram submetidos à análise de variância pelo teste $\mathrm{F}$, seguido por análises de regressões (utilizando as médias de cada tratamento) para o fator quantitativo e teste de Tukey para o fator qualitativo. A escolha dos modelos baseou-se na significância estatística (teste F), no ajuste do coeficiente de determinação $\left(\mathrm{R}^{2}\right)$ e no significado biológico do modelo.

\section{Resultados e Discussão}

Houve interação entre os fatores herbicidas e doses dos mesmos, para as variáveis, controle do arroz-vermelho e a intoxicação ao arroz cultivado.

Para a avaliação efetuada aos 14 dias após aplicação dos tratamentos (DAT) houve efeito das doses dos herbicidas no controle de arroz-vermelho. Com o incremento da dose dos produtos ocorreu maior controle da planta daninha (Figura 1). Dentre os herbicidas testados o mais eficaz foi o imazapyr + imazapic, sendo que, a partir da metade da dose recomendada $\left(73,5+24,5 \mathrm{~g} \mathrm{ha}^{-1}\right)$ ocorreu controle de $85 \%$ do arroz-vermelho chegando a mais de 91\% no dobro da dose (Figura 1 e Tabela 1). Observou-se ainda que a mistura formulada de imazethapyr + imazapic aplicada na metade da dose recomendada proporcionou controle de $58 \%$ e mesmo no dobro da dose, o máximo de controle foi de $73 \%$, porcentagem considerada ineficaz para o controle de arrozvermelho (Villa et al., 2006).

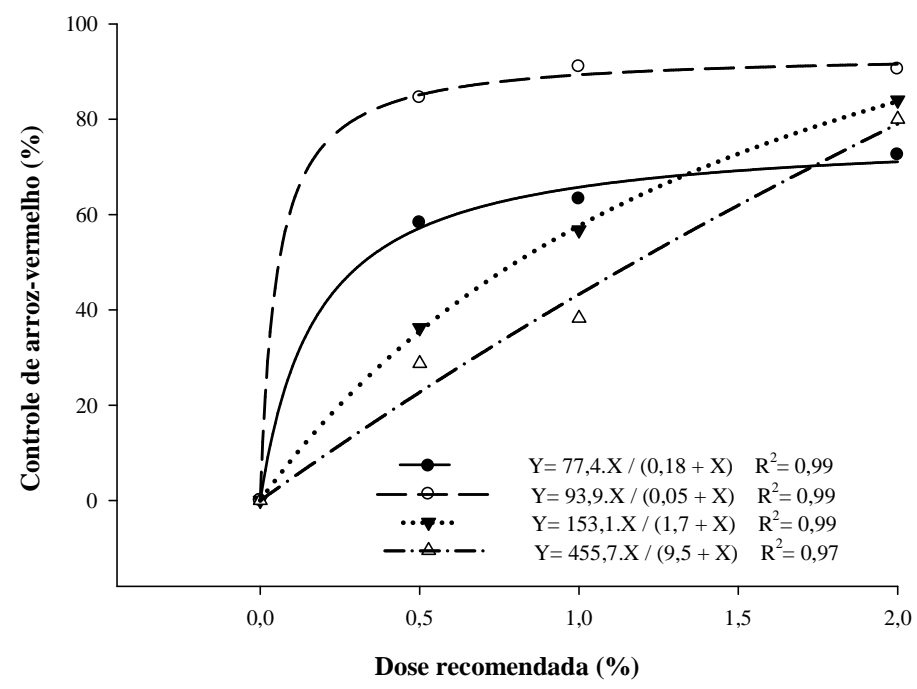

Figura 1. Controle (\%) de arroz-vermelho, aos 14 dias após a aplicação dos tratamentos (DAT), em função da porcentagem da dose recomendada dos herbicidas imazethapyr + imazapic $\bullet$ - 0; 50; 100 e 200\%), imazapyr + imazapic ( - 0; 50; 100 e 200\%), ( $\boldsymbol{\nabla}-0 ; 50 ; 100$ e 200\%) e imazapic $(\Delta-0 ; 50 ; 100$ e 200\%) à cultura do arroz irrigado. Itaqui-RS, 2011.

Os herbicidas imazethapyr e imazapic ocasionaram controles máximos de 79 e $80 \%$ respectivamente, nas maiores doses avaliadas aos 14 DAT (Tabela 1). Considerando que uma planta $\mathrm{m}^{-2}$ de arroz-vermelho ocasiona uma redução da produtividade de grãos do arroz cultivado de 2,1\% (Pantone \& Baker, 1991), não se considera eficiente e nem recomendável 
à aplicação desses produtos para controle da planta daninha em questão.

Tabela 1. Controle (\%) de arroz-vermelho aos 14 dias após a aplicação dos tratamentos (DAT) em função de herbicidas aplicados em diferentes doses. Itaqui-RS, 2011.

\begin{tabular}{lcccc}
\hline \multirow{2}{*}{ Herbicidas } & \multicolumn{4}{c}{ Dose recomendada (\%) } \\
\cline { 2 - 5 } & 0 & 50 & 100 & 200 \\
\hline Imazethapyr + imazapic & $0,0 \mathrm{a}$ & $58,0 \mathrm{~b}$ & $53,0 \mathrm{~b}$ & $73,0 \mathrm{~b}$ \\
imazapyr + imazapic & $0,0 \mathrm{a}$ & $85,0 \mathrm{a}$ & $91,0 \mathrm{a}$ & $90,5 \mathrm{a}$ \\
Imazethapyr & $0,0 \mathrm{a}$ & $33,8 \mathrm{c}$ & $56,3 \mathrm{~b}$ & $79,0 \mathrm{ab}$ \\
Imazapic & $0,0 \mathrm{a}$ & $35,0 \mathrm{c}$ & $38,3 \mathrm{c}$ & $80,0 \mathrm{ab}$ \\
\hline CV $(\%)$ & & \multicolumn{3}{c}{16,24}
\end{tabular}

${ }^{1}$ Médias seguidas de mesmas letras minúsculas na coluna não diferem entre si pelo teste de Tukey a 5\% de probabilidade.

$\mathrm{Na}$ avaliação de controle efetuada aos 28 DAT observou-se tendência similar ao encontrado na primeira avaliação. Novamente a mistura formulada de imazapyr + imazapic independente da dose testada sobressaiu-se aos demais produtos avaliados com aumento do controle, decorrente do incremento das doses dos herbicidas, seguida de imazethapyr + imazapic (Figura 2). Destaca-se ainda que os herbicidas não registrados para a cultura (imazethapyr e imazapic) apresentaram menor índice de controle em todas as doses testadas comparados com a avaliação efetuada aos 14 DAT.

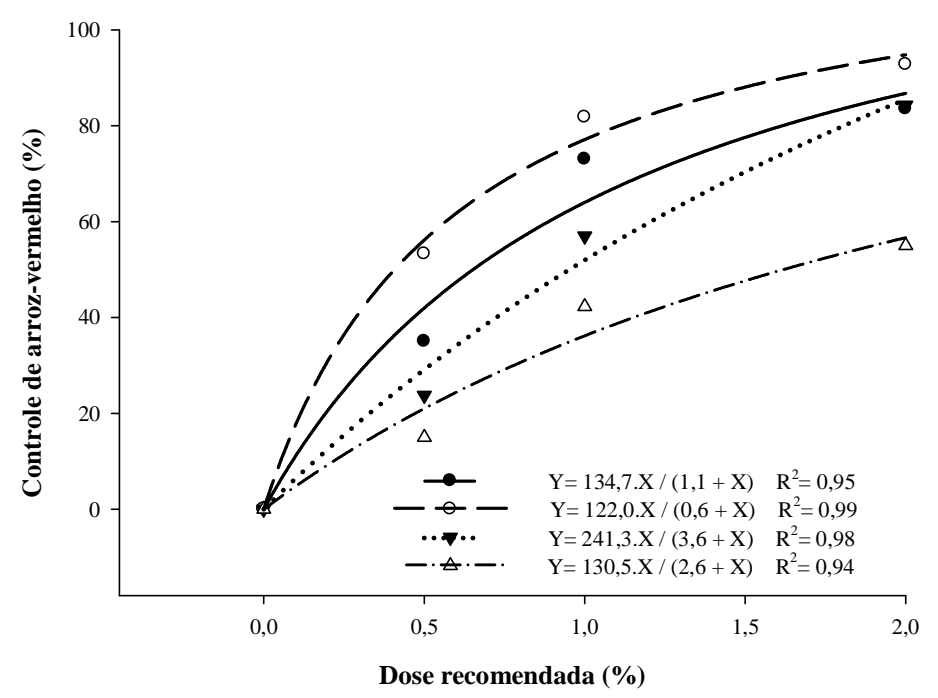

Figura 2. Controle (\%) de arroz-vermelho, aos 28 dias após a aplicação dos tratamentos (DAT), em função da porcentagem da dose recomendada dos herbicidas imazethapyr + imazapic- $(\bullet-0$; 50; 100 e 200\%), imazapyr + imazapic ( $-0 ; 50 ; 100$ e 200\%), imazethapyr ( $\boldsymbol{\nabla}-0 ; 50 ; 100$ e $200 \%)$ e imazapic ( $\Delta$ - 0; 50; 100 e 200\%) à cultura do arroz irrigado. Itaqui-RS, 2011.

Comparando-se os herbicidas entre si aos 28 DAT (Tabela 2) observou-se que o imazapyr + imazapic em meia $(36,75+12,25 \mathrm{~g}$ $\mathrm{ha}^{-1}$ ou $\left.50 \%\right)$ e na dose recomendada $(73,5+$ $24,5 \mathrm{~g} \mathrm{ha}^{-1}$ ou $100 \%$ ) apresentou os melhores controles em relação aos demais tratamentos. Já no dobro da dose somente o imazapic apresentou controle inferior aos demais, não podendo ser recomendado para o controle de arroz-vermelho já que a percentagem de 
controle foi de somente $48 \%$, muito abaixo do 2006). mínimo necessário que é de $80 \%$ (Villa et al.,

Tabela 2. Controle (\%) de arroz-vermelho aos 28 dias após a aplicação dos tratamentos (DAT) em função de herbicidas aplicados em diferentes doses. Itaqui-RS, 2011.

\begin{tabular}{lcccc}
\hline \multirow{2}{*}{ Herbicidas } & \multicolumn{4}{c}{ Dose recomendada (\%) } \\
\cline { 2 - 5 } & 0 & 50 & 100 & 200 \\
\hline Imazethapyr + imazapic & $0,0 \mathrm{a}^{1}$ & $30,0 \mathrm{~b}$ & $61,0 \mathrm{~b}$ & $83,5 \mathrm{a}$ \\
Imazapyr + imazapic & $0,0 \mathrm{a}$ & $48,8 \mathrm{a}$ & $81,8 \mathrm{a}$ & $92,8 \mathrm{a}$ \\
Imazethapyr & $0,0 \mathrm{a}$ & $18,8 \mathrm{c}$ & $55,0 \mathrm{~b}$ & $84,3 \mathrm{a}$ \\
Imazapic & $0,0 \mathrm{a}$ & $7,5 \mathrm{~d}$ & $42,3 \mathrm{c}$ & $55,0 \mathrm{~b}$ \\
\hline
\end{tabular}

CV (\%) 14,28

${ }^{1}$ Médias seguidas de mesmas letras minúsculas na coluna não diferem entre si pelo teste de Tukey a $5 \%$ de probabilidade.

A mistura formulada comercialmente composta por imazethapyr + imazapic nas doses de $75+25$ e $150+50 \mathrm{~g} \mathrm{ha}^{-1}$ apresentaram controles de 81 e $96 \%$, respectivamente (Figura 3; Tabela 3) na última avaliação em pré-colheita da cultura. As aplicações efetuadas no experimento foram únicas, mas existem relatos de orizicultores da Fronteira Oeste do RS que as aplicações sequienciais desses produtos apresentam melhores efeitos se comparado às aplicações únicas. No entanto, resultados semelhantes a esses foram relatados por Fleck et al. (2003) e Pinto et al. (2003) onde os autores não encontraram diferenças de controle entre aplicações únicas ou sequienciais utilizando o herbicida imazethapyr + imazapic para o controle do arroz-vermelho. Ao se aplicar o dobro da dose recomendada de imazapyr + imazapic observou-se controle de $95 \%$. O imazethapyr foi o único, dentre os produtos não registrados, que no dobro da dose recomendada ocasionou controle de $90,8 \%$.

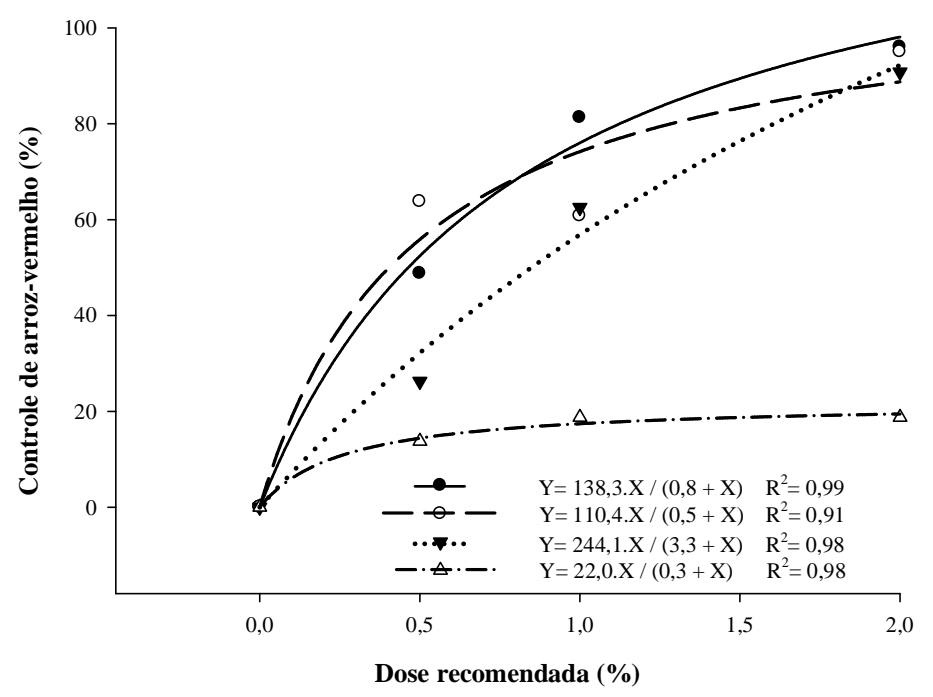

Figura 3. Controle (\%) de arroz-vermelho na pré-colheita do arroz irrigado, em função da porcentagem da dose recomendada dos herbicidas imazethapyr + imazapic $(\bullet-0 ; 50 ; 100$ e $200 \%)$, imazapyr + imazapic ( o - 0;50; 100 e 200\%), imazethapyr ( $\boldsymbol{\nabla}-0 ; 50 ; 100$ e $200 \%)$ e imazapic $(\Delta-0 ; 50 ; 100$ e 200\%) à cultura do arroz irrigado. Itaqui-RS, 2011. 
Para todos os herbicidas testados, com incremento das doses melhor foi o controle do arroz-vermelho, exceto para o imazapic que não apresentou bom controle com acréscimo das doses (Figura 3). Na dose recomendada observou-se que o imazethapyr + imazapic apresentou melhor controle do arroz-vermelho do que os demais produtos. Já no dobro da dose, novamente o único herbicida que não foi eficiente ou o que menor controle apresentou em relação aos demais foi o imazapic (Tabela 3).

Tabela 3. Controle (\%) de arroz-vermelho na pré-colheita em função de herbicidas aplicados em diferentes doses. Itaqui-RS, 2011.

\begin{tabular}{lcccc}
\hline \multirow{2}{*}{ Herbicidas } & \multicolumn{4}{c}{ Controle na pré-colheita } \\
\cline { 2 - 5 } & 0 & 50 & 100 & 200 \\
\cline { 2 - 4 } & $0,0 \mathrm{a}$ & $48,8 \mathrm{~b}$ & $81,3 \mathrm{a}$ & $96,0 \mathrm{a}$ \\
Imazethapyr + imazapic & $0,0 \mathrm{a}$ & $63,7 \mathrm{a}$ & $60,8 \mathrm{~b}$ & $95,0 \mathrm{a}$ \\
Imazapyr + imazapic & $0,0 \mathrm{a}$ & $26,3 \mathrm{~b}$ & $62,5 \mathrm{~b}$ & $90,8 \mathrm{a}$ \\
Imazethapyr & $0,0 \mathrm{a}$ & $13,8 \mathrm{c}$ & $18,8 \mathrm{c}$ & $18,8 \mathrm{~b}$ \\
Imazapic & \multicolumn{4}{c}{16,92} \\
\hline CV (\%) & \multicolumn{4}{c}{} \\
I Médias seguidas de mesmas letras minúsculas na coluna não diferem entre si pelo teste de Tukey a 5\% de \\
probabilidade
\end{tabular}

Os dados relativos à seletividade dos herbicidas sobre a cultivar Puitá Inta-CL estão representados nas Figuras 4, 5 e 6. Para todos os produtos avaliados observou-se que $o$ aumento da dose incrementou os índices de intoxicação à cultura do arroz. A mistura formulada comercialmente composta de imazapyr + imazapic demonstrou os maiores sintomas de intoxicação, com valor médio próximo a $12 \%$ (na metade da dose recomendada), seguida do imazethapyr + imazapic e imazapic (Figura 4) aos 7 DAT. Convém destacar, que ao se aplicar 49,00 $\mathrm{g} \mathrm{ha}^{-1}$ de imazapic os sintomas de intoxicação praticamente se equivaleram a imazapyr + imazapic.

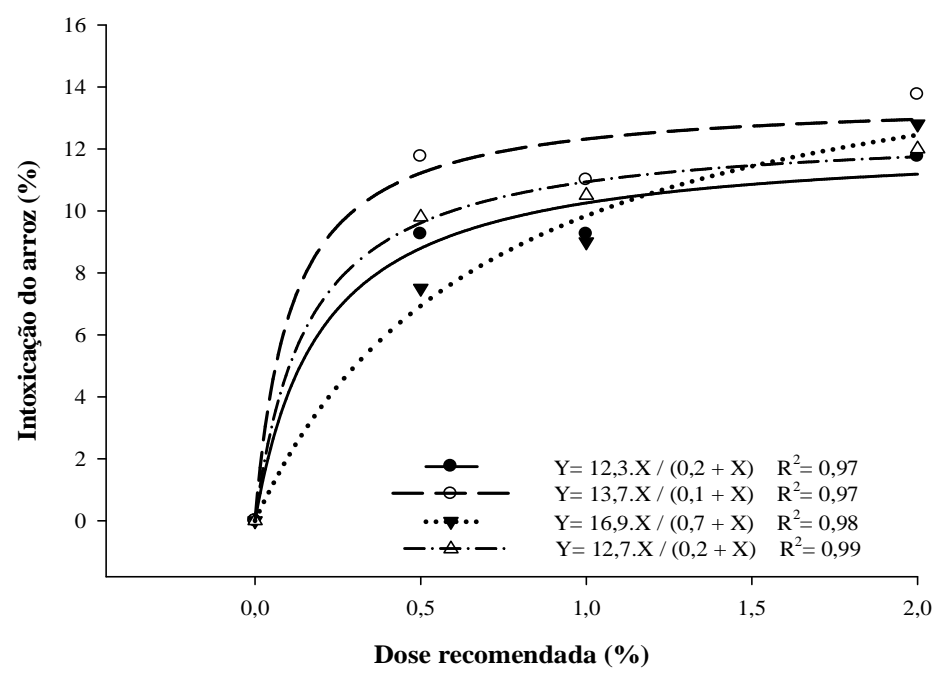

Figura 4. Intoxicação do arroz (\%), aos 7 dias após a aplicação dos tratamentos (DAT), em função da porcentagem da dose recomendada dos herbicidas imazethapyr + imazapic $(\bullet-0 ; 50$; 100 e 200\%), imazapyr + imazapic (o - 0; 50; 100 e 200\%), imazethapyr ( $\boldsymbol{\nabla}-0 ; 50 ; 100$ e $200 \%)$ e imazapic ( $\Delta$ - 0; 50; 100 e 200\%) à cultura do arroz irrigado. Itaqui-RS, 2011. 
Ao se comparar os herbicidas entre si em cada dose observam-se similaridades quanto a intoxicação das plantas de arroz aos 7 DAT, ou seja, não houve diferença entre os produtos independentemente ao se aplicar a metade, a recomendada ou o dobro da dose, exceto para o imazethapyr em $50 \%$ da dose recomendada que demonstrou menor intoxicação (Tabela 4).

Tabela 4. Intoxicação do arroz (\%) aos 7 dias após a aplicação dos tratamentos (DAT) em função de herbicidas aplicados em diferentes doses. Itaqui-RS, 2011.

\begin{tabular}{lcccc}
\hline \multicolumn{5}{c}{ Intoxicação aos 7 DAT } \\
\hline \multirow{2}{*}{ Herbicidas } & 0 & 50 & 100 & 200 \\
\cline { 2 - 5 } & $0,0 \mathrm{a}^{1}$ & $9,3 \mathrm{ab}$ & $9,3 \mathrm{a}$ & $11,8 \mathrm{a}$ \\
Imazethapyr + imazapic & $0,0 \mathrm{a}$ & $11,8 \mathrm{a}$ & $11,0 \mathrm{a}$ & $13,8 \mathrm{a}$ \\
Imazapyr + imazapic & $0,0 \mathrm{a}$ & $7,5 \mathrm{~b}$ & $9,0 \mathrm{a}$ & $12,8 \mathrm{a}$ \\
Imazethapyr & $0,0 \mathrm{a}$ & $9,8 \mathrm{ab}$ & $10,5 \mathrm{a}$ & $12,0 \mathrm{a}$ \\
Imazapic & & &
\end{tabular}

CV (\%) 30,84

${ }^{1}$ Médias seguidas de mesmas letras minúsculas na coluna não diferem entre si pelo teste de Tukey a $5 \%$ de probabilidade.

$\mathrm{Na}$ avaliação de intoxicação efetuada aos 14 DAT observou-se que os sintomas diminuíram em relação à primeira avaliação, para todos os herbicidas testados (Figura 5), no entanto, foi observada a mesma tendência da avaliação efetuada aos 7 DAT em relação aos herbicidas, imazethapyr + imazapic, imazapyr + imazapic e imazapic, mais tóxicos à cultura.

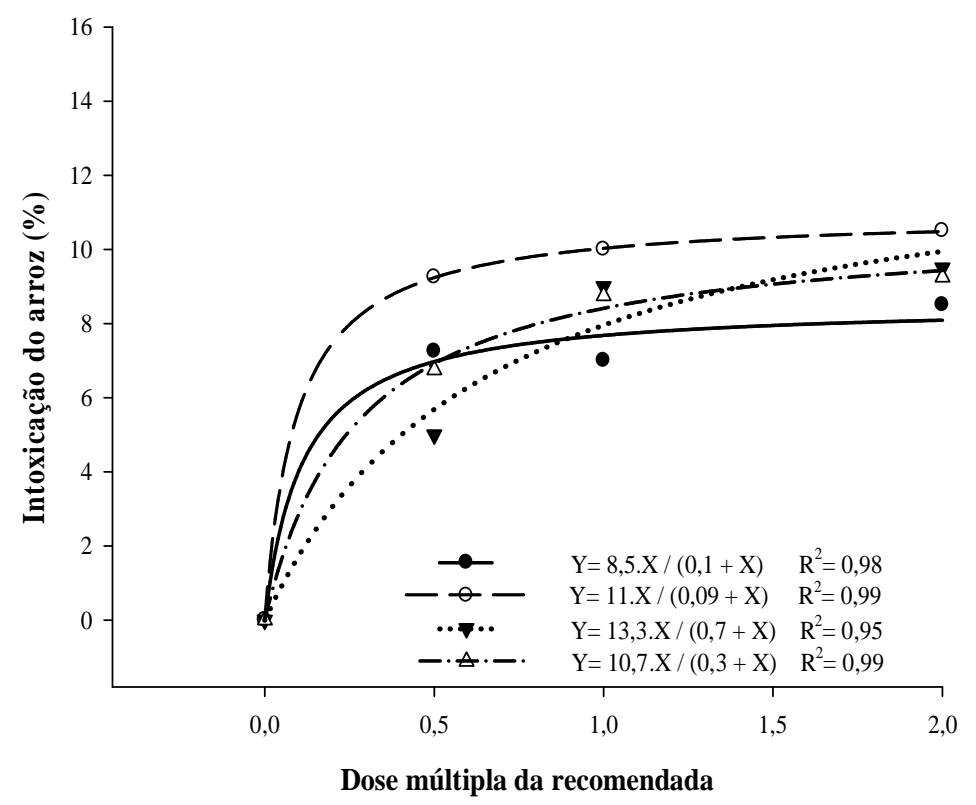

Figura 5. Intoxicação do arroz (\%), aos 14 dias após a aplicação dos tratamentos (DAT), em função da porcentagem da dose recomendada dos herbicidas imazethapyr + imazapic $(\bullet-0 ; 50$; 100 e 200\%), imazapyr + imazapic ( - 0; 50; 100 e 200\%), imazethapyr ( $\boldsymbol{\nabla}-0 ; 50 ; 100$ e $200 \%)$ e imazapic ( $\Delta$ - 0; 50; 100 e 200\%) à cultura do arroz irrigado. Itaqui-RS, 2011. 
Os resultados demonstram que aos 14 DAT ocorreu redução da intoxicação das plantas de arroz se comparada com os 7 DAT. O imazapic + imazapyr ocasionou $10 \%$ de intoxicação com 50 ou $100 \%$ da dose recomendada. Com relação à metade da dose dos herbicidas imazethapyr + imazapic e o imazapic se igualaram com $7,3 \%$, seguidos do imazethapyr com percentual inferior de $5 \%$. Com o dobro da dose recomendada, os herbicidas não diferenciaram estatisticamente entre si (Tabela 5).

Tabela 5. Intoxicação do arroz (\%) aos 14 dias após a aplicação dos tratamentos (DAT) em função de herbicidas aplicados em diferentes doses. Itaqui-RS, 2011.

\begin{tabular}{lcccc}
\hline \multicolumn{5}{c}{ Intoxicação aos 14 DAT } \\
\hline \multirow{4}{*}{ Herbicidas } & 0 & 50 & 100 & 200 \\
\cline { 2 - 5 } & $0,0 \mathrm{a}$ & $7,3 \mathrm{ab}$ & $7,0 \mathrm{~b}$ & $8,5 \mathrm{a}$ \\
Imazethapyr+imazapic & $0,0 \mathrm{a}$ & $10,0 \mathrm{a}$ & $10,0 \mathrm{a}$ & $10,5 \mathrm{a}$ \\
Imazapyr + imazapic & $0,0 \mathrm{a}$ & $5,0 \mathrm{~b}$ & $9,5 \mathrm{ab}$ & $9,5 \mathrm{a}$ \\
Imazethapyr & $0,0 \mathrm{a}$ & $7,3 \mathrm{ab}$ & $8,8 \mathrm{ab}$ & $9,0 \mathrm{a}$ \\
Imazapic & \multicolumn{5}{c}{25,18} \\
\hline CV (\%) & \multicolumn{5}{c}{} \\
I. Médias seguidas de mesmas letras minúsculas na coluna não diferem entre si pelo teste de Tukey a $5 \%$ de \\
probabilidade.
\end{tabular}

Aos 21 DAT percebe-se que as injúrias ao arroz praticamente desapareceram, com índice máximo de $4 \%$ de intoxicação ocasionada pelos herbicidas imazethapyr + imazapic e imazapy + imazapic, seguidos de imazethapyr e imazapic com 3,5\%, nas maiores doses aplicadas sobre à cultivar Puitá Inta-CL (Figura 6; Tabela 6). Isso demonstra que a cultura conseguiu recuperar-se dos sintomas de injúrias provocadas pelos herbicidas, pertencentes ao grupo químico das imidazolinonas, inibidores da enzima acetolactato sintetase (ALS), mesmo aqueles que não são recomendados. A partir dos 28 DAT não se constatou sintomas de injúrias sobre as plantas de arroz (dados não apresentados), desaparecendo por completo com o surgimento de novas folhas.

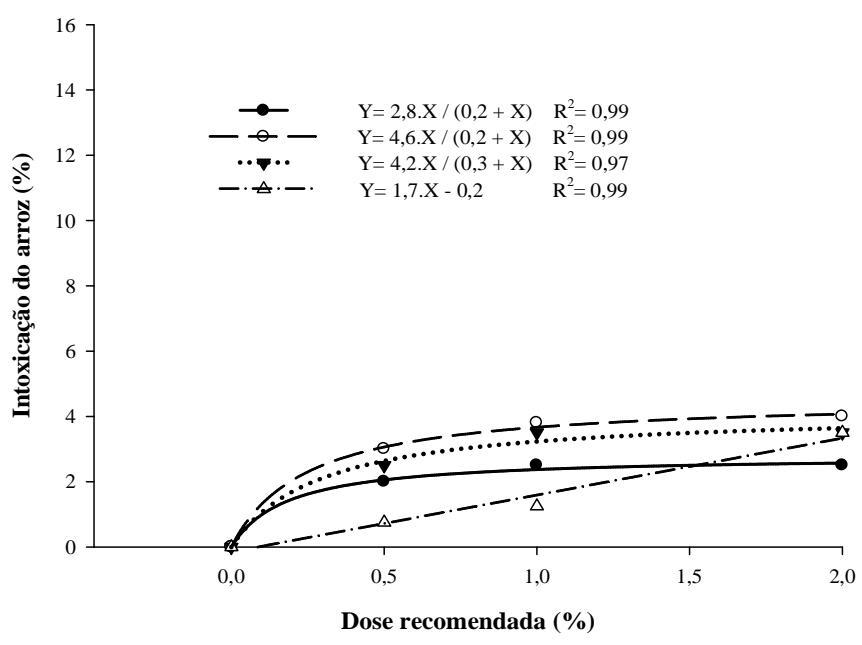

Figura 6. Intoxicação do arroz (\%), aos 21 dias após a aplicação dos tratamentos (DAT), em função da porcentagem da dose recomendada dos herbicidas imazethapyr + imazapic $(\bullet-0 ; 50$; 100 e $200 \%$ ), imazapyr + imazapic ( $-0 ; 50 ; 100$ e 200\%), imazethapyr ( $\mathbf{\nabla}-0 ; 50 ; 100$ e $200 \%)$ e imazapic ( $\Delta-0 ; 50 ; 100$ e 200\%) à cultura do arroz irrigado. Itaqui-RS, 2011. 
Os herbicidas não demonstraram diferença entre si na última avaliação de intoxicação efetuada aos 21 DAT independentemente da dose aplicada (Tabela 6). No entanto, o incremento das doses de todos os produtos ocasionaram o aumento dos índices de intoxicação à cultura do arroz irrigado na última avaliação, independentemente dos herbicidas serem ou não registrados para a cultura.

Tabela 6. Intoxicação do arroz (\%) aos 21 dias após a aplicação dos tratamentos (DAT) em função de herbicidas aplicados em diferentes doses. Itaqui-RS, 2011.

\begin{tabular}{lcccc}
\hline \multirow{2}{*}{ Herbicidas } & \multicolumn{4}{c}{ Intoxicação aos 21 DAT } \\
\cline { 2 - 5 } & 0 & 50 & 100 & 200 \\
\hline Imazethapyr + imazapic & $0,0 \mathrm{a}^{1}$ & $2,0 \mathrm{a}$ & $3,0 \mathrm{a}$ & $4,0 \mathrm{a}$ \\
Imazapyr + imazapic & $0,0 \mathrm{a}$ & $2,5 \mathrm{a}$ & $3,8 \mathrm{a}$ & $4,0 \mathrm{a}$ \\
Imazethapyr & $0,0 \mathrm{a}$ & $2,5 \mathrm{a}$ & $3,5 \mathrm{a}$ & $3,5 \mathrm{a}$ \\
Imazapic & $0,0 \mathrm{a}$ & $1,0 \mathrm{a}$ & $1,5 \mathrm{a}$ & $3,5 \mathrm{a}$ \\
\hline CV (\%) & \multicolumn{5}{c}{74,53} \\
I' Médias seguidas de mesmas letras minúsculas na coluna não diferem entre si pelo teste de Tukey a $5 \%$ de \\
probabilidade.
\end{tabular}

\section{Conclusões}

Os resultados permitem concluir que as misturas formuladas comercialmente compostas de imazapyr + imazapic a partir da dose recomendada $\left(73,5+24,5 \mathrm{~g} \mathrm{ha}^{-1}\right)$ e de imazethapyr + imazapic no dobro da dose recomendada $\left(150+50 \mathrm{~g} \mathrm{ha}^{-1}\right)$ foram eficientes para o controle de arroz-vermelho em arroz irrigado em aplicação única. Essas mesmas misturas também foram seletivas a cultivar Puitá Inta-CL, independentemente das doses aplicadas dos produtos. Os herbicidas não registrados para o controle de arroz-vermelho imazethapyr e imazapic não apresentam controle satisfatório da planta daninha.

\section{Agradecimentos}

Ao Conselho Nacional de Desenvolvimento Científico e Tecnológico (CNPq) pelas concessões de bolsas de iniciação científica ao terceiro e sétimo autores e auxílio financeiro (processo n.: n.483564/2010-9) a Leandro Galon para execução do trabalho e à Fundação de Amparo à Pesquisa do Estado do Rio Grande do Sul
(FAPERGS) pela concessão de bolsa de iniciação científica ao segundo autor.

\section{Referências}

AGOSTINETTO, D. et al. Arroz vermelho: ecofisiologia e estratégias de controle. Ciência Rural, v.31, n.2, p.341-349, 2001.

BURGOS, N.R. et al. Red rice (Oryza sativa) status after 5 years of imidazolinone-resistant rice technology in Arkansas. Weed Technology, v.22, n.1, p.200-208, 2008.

CONAB. Companhia Nacional de Abastecimento. Arroz - Brasil. Série Histórica de: área, produtividade e produção. Disponível em: http://www.conab.gov.br. Acesso em: 20/03/2012.

COX, C. Imazapyr: herbicide factsheet. Journal of Pesticide Reform Imazapyr, v.16, n.3, p.16-20, 1996.

CROUGHAN, T.P. Arroz resistente a herbicidas imidazolinonas. United States Patent, n.5,773,704.0019313, 12 jul. 1998; 25 maio 2002.

EMBRAPA - EMPRESA BRASILEIRA DE PESQUISA AGROPECUÁRIA. Centro 
Nacional de Pesquisa Agropecuária de Solos. Sistema brasileiro de classificação de solos. Rio de Janeiro: 2006. 412 p.

FLECK, N.G. et al. Controle químico seletivo de angiquinho e arroz-vermelho em arroz irrigado utilizando o sistema Clearfield®. In: CONGRESSO BRASILEIRO DE ARROZ IRRIGADO, 43.; REUNIÃO DA CULTURA DE ARROZ IRRIGADO, 25.; 2003, Balneário Camboriú - SC. Anais... Itajaí: EPAGRI, 2003. p.465-467.

GOETZ, A.J. Degradation and field persistence of imazethapyr. Weed Science, v.38, n.4/5, p.421-428, 1990.

GRYMES, C.F. Response of soybean (Glycine max) and Rice (Oryza sativa) in rotation to AC 263222. Weed Tecnology, v.9, n.3, p.504-511, 1995.

LOUX, M.M. et al. Availability and persistence of imazaquin, imazethapyr and clomazone in soil. Weed Science, v.37, n.2, p.259-267, 1989.

LOUX, M.M.; REESE, K.D. Effect of soil $\mathrm{pH}$ on adsorption and persistence of imazaquin. Weed Science, v.40, n.3, p.490-496, 1993.

MARCHESAN, E. et al. Arroz tolerante a imidazolinonas: banco de sementes de arroz vermelho e fluxo gênico. Planta Daninha, v.29, n.esp., p.1099-1105, 2011.

MENEZES, V.G. Manejo de arroz vermelho através do tipo de arranjo de plantas de arroz irrigado no sistema de cultivo mínimo. 1996. 76p. Dissertação (Mestrado em Agronomia-Fitotecnia) - Programa de Pósgraduação em Agronomia, Universidade Federal do Rio Grande do Sul, Porto Alegre, 1996.

MENEZES, V.G. et al. Arroz-vermelho (Oryza sativa) resistente aos herbicidas imidazolinonas. Planta Daninha, v.27, n. esp., p.1047-1052, 2009.
OTTIS, B.V. et al. Rice yield and quality as affected by cultivar and red rice (Oryza sativa) density. Weed Science, v.53, n.4, p.499-504, 2005 .

PANTONE, D. J.; BAKER, J. B. Reciprocal yield analysis of red rice (Oryza sativa) competition in cultivated rice. Weed Science, v.39, n.1, p.42-47, 1991.

PINTO, J.J.O. et al. Controle de arroz-daninho através do sistema de manejo Clearfield®. In: CONGRESSO BRASILEIRO DE ARROZ IRRIGADO, 43.; REUNIÃO DA CULTURA DE ARROZ IRRIGADO, 25.; 2003, Balneário Camboriú - SC. Anais... Itajaí: EPAGRI, 2003. p.468-470.

PINTO, J.J.O. et al. Milho (Zea mays) como espécie bioindicadora da atividade residual de (imazethapyr+imazapic). Planta Daninha, v.27, n. esp., p.1005-1014, 2009a.

PINTO, J.J.O. et al. Atividade residual de (imazethapyr+imazapic) para sorgo granífero (Sorghum bicolor) semeado em rotação com o arroz irrigado. Planta Daninha, v.27, n. esp., p. 1015-1024, 2009b.

PINTO, J.J.O. et al. Atividade residual de (imazethapyr+imazapic) sobre azevém anual (Lolium multiflorum), semeado em sucessão ao arroz irrigado, sistema Clearfield ${ }^{\circledR}$. Planta Daninha, v.27, n.3, p.609-619, 2009c.

RIZZARDI, M.A. et al. Resistência de plantas aos herbicidas inibidores da acetolactato sintase. Planta Daninha, v.20, n.1, p.149-158, 2002.

ROSO, A.C. et al. Bioensaios para diagnóstico da resistência aos herbicidas imidazolinonas em arroz. Planta Daninha, v.28, n.2, p.411419, 2010.

SANCHEZ-OLQUIN, E. et al. Vegetative and reproductive development of Costa Rican weedy rice compared with commercial rice (Oryza sativa). Planta Daninha, v.25, n.1, p.13-23, 2007. 
SANTOS, F.M. et al. Controle químico de arroz-vermelho na cultura do arroz irrigado. Planta Daninha, v.25, n.2, p.405-412, 2007.

SANTOS, G. et al. Uso do novo sistema Clearfield $^{\circledR}$ na cultura do girassol para o controle de plantas daninhas dicotiledôneas. Planta Daninha, v.30, n.2, p.359-365, 2012.

SOCIEDADE SUL-BRASILEIRA DE ARROZ IRRIGADO - SOSBAI. Arroz irrigado: recomendações técnicas da pesquisa para o Sul do Brasil. Bento Gonçalves: SOSBAI, 2010. 188p.

VILLA, S.C.C. et al. Controle de arrozvermelho em dois genótipos de arroz (Oryza sativa) tolerantes a herbicidas do grupo das imidazolinonas. Planta Daninha, v.24, n.3, p.549-555, 2006. 